\title{
Facebook como herramienta de marketing/comunicación para la atracción de estudiantes internacionales: un análisis de Chile y España
}

\section{Facebook as a marketing/communication tool for international students attraction: an analysis of Chile and Spain}

\author{
José Luis Meza Orellana. Universidad de Valparaíso, Chile (jose.meza@uv.cl)
}

Recibido: 13-IV-2015 - Aceptado: 13-V-2015

Resumen:

Este trabajo analiza el uso de Facebook como herramienta de marketing internacional en universidades de Chile y España. Se estudió el 93\% de las universidades de ambos países mediante el método de análisis de contenido. Los resultados muestran que el uso de Páginas de Facebook corresponde, en su mayoría, a universidades con mayor matrícula internacional. Hay una relación directa entre la cantidad de estudiantes extranjeros y el uso de Fanpage como canal de comunicación de marketing. Por el contrario, los mejores desempeños corresponden a universidades con menos extranjeros. Existe una relación inversa entre el desempeño en esas páginas y la cantidad de estudiantes extranjeros, pero directa, entre el número de estudiantes y la presencia en Facebook mediante Fan Page.

Palabras clave:

Facebook; Intercambio de Estudiantes; Redes sociales; Internacionalización; Marketing Internacional.

Abstract:

This paper aim to analyze the use of Facebook as a tool of international marketing in Chilean and Spanish universities. The study cover 93\% of universities in both countries, and using the content analysis method, we can establish that the use of Fan Pages is mainly related to universities with higher number of international student enrolled. There is a direct relation between the enrollment of international students and the use of Facebook as an international marketing communication tool. On the contrary, Universities with less international enrollment seems to have better performances. There is an inverse relation between the Fan Page performance and the number of international students, and a direct one between the number of international students and the use of Fan Pages, as a way to be present in Facebook.

Keywords:

Facebook; Student Exchange; Social Networks; Internationalization; International Marketing. 


\section{Introducción}

\subsection{Comunicación de Marketing en entornos globales}

La internacionalización como parte de las funciones sustantivas de las Instituciones de Educación Superior (en adelante IES), se ha convertido en una realidad ampliamente compartida entre universidades en todo el mundo (Gacel-Ávila, 2012; Knight, 2013), asimismo, la globalización ha desencadenado, en los entornos universitarios, procesos de internacionalización sin precedentes. La intervención de los capitales privados y la aparición de acuerdos y tratados internacionales en temas educativos, impulsa la consolidación del “mercado global de la educación" (Gordan y Pop, 2013; Tofallis, 2012). En este nuevo escenario, las instituciones han dejado de competir en sus propios países, y han tenido que comenzar a buscar a sus estudiantes, sus socios para la investigación y los proyectos, e incluso, a sus financistas, en otras latitudes.

En este mercado global educativo, las universidades no están exentas de la necesidad de comunicar activamente para conseguir una posición de ventaja. Así, las IES deben comprometerse con estrategias de comunicación internacional y reorientar a una mirada más próxima al marketing, sus esfuerzos de vinculación con estudiantes. Con esta orientación, podrán alcanzar diferenciación frente a los competidores, y conseguir sus objetivos al vincularse efectivamente con sus stakeholders internacionales.

Para las compañías orientadas a los consumidores, que desarrollan campañas de marketing en social media con grandes audiencias como Facebook o Twitter, uno de los objetivos centrales es el engagement (Lee; Hosanagar; y Nair, 2014). Producto de las recientes consideraciones, que plantean que las métricas asociadas al número de seguidores no aportan una directa relación con el compromiso (engagement) alcanzado por los usuarios (Wallace et al., 2014), los esfuerzos en Social Media Marketing han cambiado; han pasado de centrarse únicamente en la atracción de nuevos seguidores, a procurar el compromiso de estos. Lee et al. (2014) ponen como ejemplo a General Motors, que pasó de destinar cerca de 10 millones de dólares en anuncios pagados en Facebook (usualmente dirigidos a captar nuevos fans), a gastar 30 millones de dólares en esfuerzos destinados a crear contenido para aumentar el compromiso de sus seguidores.

Este fenómeno no excluye a la Educación Superior. La competencia intensa y el crecimiento de la industria global de la educación, la obliga a considerar nuevas estrategias dirigidas a la atracción de estudiantes (Alzamel, 2014), particularmente estudiantes internacionales. En este sentido, y para asegurar su subsistencia en los mercados globales, las IES necesitan establecer mecanismos que faciliten el flujo de contenido hacia y desde sus stakeholders (Ross; Grace; y Shao, 2012) y de ese modo, satisfacer los crecientes requerimientos de información para la toma de decisiones entre los estudiantes internacionales.

La aparición de las redes sociales ha cambiado drásticamente la forma en que los consumidores interactúan en los mercados, especialmente en la forma en que adquieren información sobre los productos y servicios, y el modo en que los 
consumen (Hennig-Thurau et al., 2010). Fagerstrom y Ghinea (2013), citando a O'Connor y Lundstrom (2011), plantean que según variados estudios, los estudiantes prefieren obtener información de modo personal-informal, en vez de aquella entregada en un formato más formal.

La cercanía y la informalidad de las redes sociales, ofrecen una ventaja distintiva frente a otro tipo de acciones de comunicación universitaria (Reina; Fernández; y Noguer, 2012), por esta razón, muchas organizaciones las han integrado como parte de sus herramientas de comunicación y marketing. De hecho, según reportes de las compañías Burson-Marsteller (2012) y Mashable Social Media (2011), citadas por Wilcox y Kim (2012), el 75\% de las compañías en el Índice Fortune 500, están usando, al menos, una plataforma social. Concretamente, el 54\% de las listadas en las Fortune 100, usan Fan page. Así mismo el $92 \%$ de las organizaciones sin fines de lucro incluyen acceso a al menos una de estas plataformas sociales en sus páginas web.

\subsection{Comunicación de Marketing en Social Media}

Estudiantes buscando nuevas formas de conseguir información y universidades forzadas a la implementación de nuevas estrategias para difundir sus servicios, hacen que se modifique el centro de atención de la estrategia de marketing educativo, pasando de una visión centrada en la organización, a otra con foco en los estudiantes y en la creación de experiencias entre éstos y la universidad. Este fenómeno, conocido como la co-creación de valor (Grönroos, 2008), parece estar guiando el comportamiento de la comunicación de marketing en las universidades, particularmente en las acciones desplegadas en redes sociales. Por ejemplo, plataformas como Facebook, Twitter y YouTube, descansan en el contenido generado por el usuario para su atracción y retención (Hennig-Thurau et al., 2010).

Estudios empíricos muestran que las nuevas tecnologías de la información han sido cruciales para el marketing internacional de las universidades. La disponibilidad y calidad de la información las trasforman en una variable fundamental para los estudiantes al momento de elegir una universidad extranjera (Thune y Welle-Strand, 2005). Thune y Welle-Strand afirman que estas nuevas tecnologías de la información son importantes para la movilidad de estudiantes y para el reclutamiento de estudiantes extranjeros. Sus hallazgos confirman que Internet ha sido señalado como el principal sitio para la promoción y para la información de nuevos prospectos internacionales. Internet es identificado por las oficinas de relaciones internacionales como el lugar donde muchos estudiantes han conseguido información respecto de la universidad antes de matricularse en ellas.

Investigadores han demostrado que un acercamiento estratégico a la comunicación de marketing en redes sociales ayuda a construir la Marca y la Reputación (Tomše y Snoj, 2014), para aquello se hace necesario crear comunicación con significado para los usuarios objetivo. Cuando la organización conoce qué información los usuarios valoran, puede involucrarse con ellos en una conversación valiosa y duradera. La comunicación de marketing en redes sociales, ofrece posibilidades 
bastante distintas a las de los medios tradicionales (Tomše y Snoj, 2014). Colliander (2012), apunta que las redes sociales son más efectivas que los medios tradicionales en el posicionamiento de marca y en percepción de marca, dado que sus usuarios tienen la posibilidad de experimentar la comunicación de un modo bidireccional.

Las redes sociales ofrecen entonces, una gran oportunidad para reemplazar una visión pasiva de los estudiantes por una activa, en donde los postulantes son incitados a usar su iniciativa en vez de reaccionar a las actividades típicas del Marketing tradicional (Fagerstrøm y Ghinea, 2013).

Wilcox y Kyungok (2012) describen un modelo presentado por Murdough (2010) en el que se incluyen cinco fases para la planeación de una estrategia: concept, definition, design, deployment y optimization (Concepto, Definición, Diseño, Implementación y Optimización). El modelo consigna a la primera fase, la de concepto, la definición de los objetivos y medidas para los resultados deseados (Key Performance Indicators) (Kaplan; y Norton, 2007). Murdough enfatiza que es esencial para la ejecución de todo el proceso, el continuo seguimiento y medición de estos indicadores y la consecuente corrección de los mensajes para conseguir los resultados esperados.

Murdough señala además (2010), que es necesaria la evaluación continua de todo el proceso, debido a su naturaleza basada en la interacción y el compromiso de los usuarios. Pero, capturar ese compromiso e interacción en un momento estático de la conducta comunicacional es probablemente poco efectivo; de modo que es necesario incluir procesos de evaluación de variables con un carácter dinámico. Un modelo de evaluación de Redes Sociales deberá incluir, entonces, el seguimiento a lo largo del tiempo, de las siguientes variables: a) Frecuencia: busca medir la tasa de ocurrencia de las actividades de comunicación, b) el Alcance: determinado por el tamaño de la comunidad c) El compromiso de la audiencia o engagement.

El autor detalla una definición de estas variables, planteadas por Kaushik (2010):

Alcance, será el tamaño de la comunidad a la que se puede llegar a través de la actividad en redes sociales. Este factor resulta muy relevante para la difusión de los mensajes, si reconocemos la naturaleza viral de los contenidos más comúnmente difundidos y el poder que propagación que las plataformas sociales ofrecen. Un mayor número de seguidores o Fans, permitirá la mejor propagación de contenidos de manera viral, y además facilitará la recomendación de los propios seguidores a sus amigos en Facebook, con el consiguiente valor que ello representa. Los usuarios asignan gran credibilidad a las recomendaciones proporcionadas por pares, provenientes de la experiencia y en entornos menos formales.

Frecuencia, se refiere a la cantidad específica de acciones publicadas para los usuarios on line en un periodo de tiempo. Esta variable tendrá un impacto directo en la cantidad de impresiones que la organización es capaz de generar, y el interés que logre mantener entre los Fans. Una organización que genere publicaciones constantes, tanto informativas como persuasivas, tendrá más opciones de impactar a sus seguidores, y sobre todo, conseguir interactuar con ellos. 
Finalmente, el engagement, queda definido como el total de interacciones que la organización está experimentando con sus usuarios de redes sociales. La medición de esta última variable incluye información respecto de los comentarios; los "me gusta"; el número de clics a enlaces hechos en Facebook; y el número de respuestas, re tweets y clics a enlaces conseguidos en Twitter. Esta variable medirá el objetivo final de la comunicación en redes sociales, la vinculación, el compromiso de los seguidores. Medirá el momento en que la co-creación de contenido conduce a la co-creación de valor.

La gestión de la comunicación en redes sociales, como hemos visto, ha recibido bastante atención por parte de los investigadores, y es profusa la cantidad de publicaciones que hacen referencia a su gestión, medición y análisis teórico (Boyd y Ellison, 2007; Carpenter y Drezner, 2010; González-Molina y Ramos, 2014; Wilcox y Kim, 2013; Wilcox y Kim, 2012).

Una situación similar ocurre cuando se observa la internacionalización, y en particular la atracción de estudiantes extranjeros. Un número considerable de autores han procurado comprender no solo cómo actúa el proceso de toma de decisión de los estudiantes para seleccionar una universidad o país (Maringe y Carter, 2007; Mazzarol y Soutar, 2002; Wei, 2012; Wilkins, Balakrishnan y Huisman, 2012); sino además, el modo en que las universidades procuran atraerlos y transmitirles sus ventajas (Chapleo, Carrillo; y Castillo, 2011; Djerasimovic, 2014; Pinto, Guerrero Quesada y Granell, 2014).

Aun con lo anterior, en la literatura formal existen pocas referencias a la relación que pudiera haber entre el uso de herramientas de comunicación en redes sociales y el éxito de los procesos de internacionalización en las universidades, específicamente en la atracción de estudiantes extranjeros.

Esta carencia de evidencia científica nos hace plantear las interrogantes que guían esta investigación, que buscan una aproximación inicial y exploratoria al uso de la comunicación en redes sociales y la atracción de estudiantes, como la modalidad más común de internacionalización universitaria.

PI1: ¿Las universidades de Chile y España, usan sitios web específicos para ofrecer contenidos dirigidos, en particular, a stakeholders internacionales?

PI2: ¿Las universidades chilenas y españolas, están presentes en Facebook, usan esta plataforma social para su comunicación con públicos extranjeros?

PI3: ¿Las universidades están usando Facebook para la comunicación con sus públicos internacionales, y a través de qué tipo de soporte en la red social, difunden sus contenidos?

PI4: ¿Existe alguna relación entre el uso de herramientas en Facebook y el éxito en los procesos de internacionalización, especialmente en lo referido a la atracción de estudiantes extranjeros?

PI4: ¿Cuál es el desempeño de las universidades en sus acciones en Facebook y, es posible establecer una relación entre esos desempeños y la atracción de estudiantes extranjeros? 


\section{Métodos}

El objeto de estudio del presente artículo se centra en los esfuerzos de comunicación de marketing desarrollados por las universidades de Chile y España, orientados particularmente a la atracción de estudiantes extranjeros.

Para este efecto, se analizó una muestra constituida por la totalidad de las universidades del sistema de educación superior español y chileno. Tanto de las instituciones del Estado como aquellas que pertenecen a intereses privados. Mediante la revisión de sus sitios web institucionales se accedió a aquellas secciones con contenido dirigido a stakeholders internacionales (que hemos llamado "sitios internacionales"). Desde estos sitios, accedimos a las herramientas de Facebook con propósitos de internacionalización.

No todas las instituciones ofrecían un enlace directo a Facebook desde sus sitios internacionales, por lo que desarrollamos, además, búsquedas en las propias plataformas sociales y en Google, con el fin de establecer la presencia de las instituciones de la muestra.

\subsection{Muestra}

El sistema español de educación superior está constituido por 82 universidades, de las cuales únicamente 80 se encuentran impartiendo servicios académicos. De ellas, 50 son públicas y las restantes 30, de carácter privado (Gobierno de España, 2015). Para seleccionar las universidades españolas sujetas a este análisis, se consideró, únicamente, a aquellas cuyos datos de matrícula extranjera estuvieron disponibles y actualizados en el último informe de datos estadísticos del sistema universitario español. Este informe incluye información de matrícula nacional y extranjera correspondiente al periodo 2011-2012, y ofreció, únicamente, los datos de 76 universidades, de las cuales 49 tienen un carácter público y 27 son privadas.

La muestra española incluye entonces, a las universidades de España cuyos datos de matrícula estuvieron disponibles en el último informe estadístico publicado por el Ministerio de Educación, Cultura y Deporte del Gobierno de España (76) (Gobierno de España, 2013).

El sistema universitario de educación superior chileno, por su parte, está integrado por 59 universidades, de las cuales 35 corresponden a corporaciones de derecho privado o fundaciones y, por tanto, son consideradas universidades de propiedad privada. Sin embargo, 24 de ellas tienen un carácter de "servicio público" (Gobierno de Chile, 2014a) y, aunque no todas pertenecen al Estado, reciben recursos económicos de éste y se agrupan en el Consejo de Rectores de Universidades Chilenas. Estas Universidades son denominadas “Universidades Tradicionales” y, para efectos de esta investigación, serán consideradas universidades públicas. No por su propiedad, sino por sus propósitos.

De las 35 universidades privadas de Chile, incluidas en el Directorio Instituciones Educación Superior, se analizaron 31, puesto que 4 de ellas no registran matrícula los últimos dos años, ya sea porque han perdido la acreditación de calidad del

60 | n²1, pp. 55-77 | doxa.comunicación 
Consejo Nacional de Acreditación o porque se les ha retirado el reconocimiento oficial y, por tanto, la autorización para funcionar.

La muestra analizada entonces, en el caso de sistema universitario de educación superior chileno, fue de 55 instituciones.

De este modo, para un universo de 139 universidades en ambos países, se analizó una muestra de 131 instituciones, que para un nivel de confianza del 95\%, espera resultados con un margen de error del $2 \%$.

\subsection{Estrategia metodológica}

La estrategia metodológica tiene un carácter cuantitativo, y consistió en el análisis de contenidos de las herramientas web y Páginas de Facebook usadas por las instituciones de educación superior de ambos países. En total, el estudio abarcó los sitios web institucionales y los sitios específicos de temas internacionales de 131 universidades en Chile y España, que representan al 93\% de las universidades existentes en ambos países.

Considerando la penetración de las redes sociales y particularmente, la orientación al marketing de las Páginas de Facebook, se analizó el comportamiento de las universidades en esta plataforma.

2.2.1. Variables independientes para el análisis de redes sociales.

2.2.1.1. Presencia de la institución en Facebook: se analizó la existencia de soportes en la red social, que tuvieran una orientación clara a la internacionalización.

2.2.1.2. Tipo de presencia en Facebook: con el fin de establecer la orientación estratégica de su uso, se buscó determinar el tipo de soporte que cada universidad utilizó para fines de internacionalización, se estableció una diferenciación entre Perfil, Página, Grupo abierto y Grupo cerrado.

2.2.1.3. Rendimiento: producto de la específica utilidad de las Páginas de Facebook para fines de marketing, analizamos el desempeño de las IES en el uso de Fan Pages, midiendo los siguientes indicadores:

a) Alcance: determinado por el número fans, variable a la que nos referiremos como Tamaño de la Comunidad.

b) Frecuencia: consideró la cantidad total de publicaciones realizadas por la institución en una relación diaria.

c) Engagement: se refiere al número de interacciones que los receptores realizaron con los contenidos publicados en la Fan Page, ya sea por la propia institución o por algún miembro de su comunidad. El indicador que define el engagement se consigue de la división del total de interacciones, presentadas como el PTAT (People talking about that, Personas hablando de esto) sobre número total de miembros de la comunidad. (Murdough, 2010). 
El estudio consideró el número de estudiantes extranjeros matriculados en cada universidad como factor de éxito en la atracción de estudiantes extranjeros. Estos datos permitieron comparar la relación entre el uso de Fan Page como herramienta de atracción de estudiantes internacionales, y los resultados de la universidad en esta dimensión, procurando establecer una correlación entre el uso de Fan Page y mejores índices de matrícula internacional.

Para determinar el número de estudiantes extranjeros consultamos los datos estadísticos oficiales publicados tanto por el Gobierno de España (2014), como el de Chile (2014b). Los datos disponibles en España incluían información solamente hasta el periodo 2011-2012, de modo que para Chile se usó el mismo periodo, en este caso, por la diferencia de inicio del año académico, los datos de 2011.

Una vez obtenidos estos datos, se agruparon las universidades en cuartiles según número de estudiantes extranjeros matriculados y, quedaron configurados de la siguiente manera:

Cuartil 1: universidades entre 0 y 179 estudiantes extranjeros; Cuartil 2: universidades con entre 180 y 478 estudiantes extranjero; Cuartil 3: universidades con entre 479 y 1.182 estudiantes y, finalmente, el Cuartil 4, quedó integrado por las universidades que matricularon entre 1.183 y 5.220 estudiantes extranjeros (Ver Cuadro 1).

Cuadro 1. Matrícula acumulada y matrícula promedio de estudiantes extranjeros, por cuartil, del total de Universidades

\begin{tabular}{|c|c|c|}
\hline & Matrícula Acumulada & Matrícula Promedio \\
\hline Cuartil 1 & 2.274 & 71 \\
\hline Cuartil 2 & 9.905 & 300 \\
\hline Cuartil 3 & 25.666 & 778 \\
\hline Cuartil 4 & 76.740 & 2.325 \\
\hline
\end{tabular}

Fuente: Elaboración propia con datos de los Gobiernos de España y Chile

\section{Resultados}

\subsection{Sitios web internacionales}

42 de las 55 universidades chilenas poseen un sitio con información específica internacional. En España la proporción es 73 de 76.

Al desagregar esos datos entre las instituciones de carácter público y privado, observamos que, en el caso de las públicas, el $96 \%$ de las españolas tiene un sitio internacional. En Chile un 83\% mantuvo este tipo de sección. 
En las universidades privadas, en cambio, los sitios internacionales estuvieron presentes en el $71 \%$ de las instituciones de Chile y en un $96 \%$ de las españolas (Ver Cuadro 2).

Cuadro 2. Universidades que cuentan con un sitio específico de contenido para la internacionalización

\begin{tabular}{|c|c|c|c|c|c|c|}
\hline & \multicolumn{2}{|c|}{ Públicas } & \multicolumn{2}{c|}{ Privadas } & \multicolumn{2}{c|}{ TOTAL } \\
\hline & IES & $\%$ & IES & $\%$ & IES & $\%$ \\
\hline Chile & 20 & $83 \%$ & 22 & $71 \%$ & 42 & $76 \%$ \\
\hline España & 47 & $96 \%$ & 26 & $96 \%$ & 73 & $96 \%$ \\
\hline
\end{tabular}

Fuente: Elaboración propia con datos de la investigación

La presencia de este tipo de sitios puede facilitar la búsqueda de información y podría, entonces, favorecer la atracción de estudiantes internacionales. Una primera relación entre el número de estudiantes extranjeros matriculados y la presencia de sitios internacionales, demostró que existe coherencia entre el uso de este tipo de sitio y el número de estudiantes extranjeros matriculados en la universidad. En el cuartil con mayor número de estudiantes extranjeros en ambos países, el $100 \%$ de las instituciones tenía un sitio internacional, mientras que la tendencia fue bajando, en número y proporción, a medida que el volumen de estudiantes extranjeros descendió.

Cuadro 3. Universidades que sí tienen un sitio internacional como porcentaje del cuartil de matrícula internacional

\begin{tabular}{|c|c|c|}
\hline & $\mathrm{N}^{\circ}$ Universidades con un sitio internacional & Como \% en el cuartil \\
\hline Cuartil 1 & 21 & $64 \%$ \\
\hline Cuartil 2 & 29 & $88 \%$ \\
\hline Cuartil 3 & 32 & $97 \%$ \\
\hline Cuartil 4 & 33 & $100 \%$ \\
\hline
\end{tabular}

Fuente: Elaboración propia con datos de la investigación

La tendencia se repite cuando analizamos estos datos desagregados por país, considerando la representación de las universidades en cada cuartil. En ambos países, las universidades que más estudiantes extranjeros han matriculado, son las que, en mayor proporción, mantienen sitios internacionales. 
Cuadro 4. Universidades con sitios internacionales por cuartil, desagregados por país

\begin{tabular}{|c|c|c|c|c|c|c|}
\hline & \multicolumn{3}{|c|}{ CHILE } & \multicolumn{3}{c|}{ ESPAÑA } \\
\hline & $\begin{array}{c}\text { Tiene un Sitio } \\
\text { Internacional }\end{array}$ & $\begin{array}{c}\mathrm{N}^{\circ} \text { IES en el } \\
\text { Cuartil }\end{array}$ & \% del Cuartil & $\begin{array}{c}\text { Tiene un Sitio } \\
\text { Internacional }\end{array}$ & $\begin{array}{c}\mathrm{N}^{\circ} \text { IES en el } \\
\text { Cuartil }\end{array}$ & \% del Cuartil \\
\hline Cuartil 1 & 10 & 18 & $56 \%$ & 11 & 14 & $79 \%$ \\
\hline Cuartil 2 & 10 & 14 & $71 \%$ & 19 & 19 & $100 \%$ \\
\hline Cuartil 3 & 12 & 13 & $92 \%$ & 20 & 20 & $100 \%$ \\
\hline Cuartil 4 & 10 & 10 & $100 \%$ & 23 & 23 & $100 \%$ \\
\hline
\end{tabular}

Fuente: Elaboración propia con datos de la investigación

Como muestra el Cuadro 4, en Chile, las universidades pertenecientes a los cuartiles con mayor matricula internacional ( 3 y 4), en promedio, el $96 \%$ posee un sitio internacional. En España en tanto, todas las universidades agrupadas en los cuartiles 2, 3 y 4, es decir aquellas con mayor matrícula internacional, tienen un sitio web internacional.

\subsection{Presencia en Facebook}

En España, poco más de la mitad de las instituciones analizadas tiene presencia en Facebook, un 53\% del total. De estas, 29 universidades son públicas (59\% del total de esa categoría) y 11 son privadas (41\% del total de la categoría).

Los datos para Chile, en cambio, demostraron que el 38\% de las instituciones públicas están presentes en Facebook; mientras que, apenas, un $23 \%$ de las privadas usa la plataforma social. Las universidades chilenas que están presentes en esta red social, representan únicamente el 29\% de la muestra analizada en ese país (Ver Cuadro 5).

Cuadro 5. Universidades con presencia en Facebook, según país y tipo de institución

\begin{tabular}{|c|c|c|c|c|c|c|}
\hline & \multicolumn{2}{|c|}{ TOTAL } & \multicolumn{2}{c|}{ PÚBLICA } & \multicolumn{2}{c|}{ PRIVADA } \\
\hline & $\mathrm{N}^{\circ}$ IES & \% del Total & $\mathrm{N}^{\circ}$ IES & \% de Públicas & $\mathrm{N}^{\circ}$ IES & \% de Privadas \\
\hline CHILE & 16 & $29 \%$ & 9 & $38 \%$ & 7 & $23 \%$ \\
\hline ESPAÑA & 40 & $53 \%$ & 29 & $59 \%$ & 11 & $41 \%$ \\
\hline
\end{tabular}

Fuente: Elaboración propia con datos de la investigación

Si se expresan los datos según los cuartiles de matrícula extranjera, nuevamente la tendencia es consistente. En el cuartil de mayor matrícula internacional son más las universidades presentes en Facebook con objetivos de internacionalización, aunque apenas alcanzan un 52\%. La cifra desciende en el resto de los cuartiles de menor matrícula internacional. En

$64 \mid n^{\circ} 21$, pp. 55-77 | doxa.comunicación 
resumen, nuevamente hay una relación directamente proporcional entre las universidades presentes en Facebook y el número de matriculados internacionales (Ver Cuadro 6).

Cuadro 6. Universidades con presencia en Facebook. Número y porcentaje del cuartil

\begin{tabular}{|c|c|c|}
\hline & $\mathrm{N}^{\circ}$ de IES & Porcentaje de las IES del Cuartil. \\
\hline Cuartil 1 & 10 & $31 \%$ \\
\hline Cuartil 2 & 13 & $39 \%$ \\
\hline Cuartil 3 & 16 & $48 \%$ \\
\hline Cuartil 4 & 17 & $52 \%$ \\
\hline
\end{tabular}

Fuente: Elaboración propia con datos de la investigación

Los resultados mostraron que las universidades que están presente en Facebook, lo hacen mediante diferentes soportes, usan Fan Pages, Perfiles, Grupos cerrados y Grupos abiertos.

Solo las páginas de Facebook tienen un foco directo en marketing, y permiten la medición de indicadores de resultado. Creemos que el uso de otras alternativas podría estar demostrando una incomprensión de la utilidad de páginas de Facebook como herramientas de comunicación de marketing y el desconocimiento de las ventajas de su uso con fines de atracción de estudiantes.

Así, en el Cuadro 7 se puede observar que solo el $44 \%$ de las universidades chilenas presentes en Facebook, lo hace mediante una Fan Page. En tanto, igual número lo hace mediante un perfil.

En España, en cambio, el 60\% de las universidades presentes en la red usa Fan Page como herramienta de marketing. Mientras, solo un 23\% se inclina por el uso Perfiles y un 18\%, por Grupos.

Cuadro 7. Tipo de soporte utilizado en Facebook, como porcentaje del total de instituciones con presencia en la Red

\begin{tabular}{|c|c|c|c|c|}
\hline & \multicolumn{2}{|c|}{ CHILE } & \multicolumn{2}{c|}{ ESPAÑA } \\
\hline & $\mathrm{N}^{\circ}$ IES & \% del Total & $\mathrm{N}^{\circ}$ IES & \% del Total \\
\hline Fan Page & 7 & $44 \%$ & 24 & $60 \%$ \\
\hline Perfil & 7 & $44 \%$ & 9 & $23 \%$ \\
\hline Grupo cerrado & 2 & $12 \%$ & 1 & $15 \%$ \\
\hline Grupo abierto & 0 & $0 \%$ & $3 \%$ \\
\hline
\end{tabular}

Fuente: Elaboración propia con datos de la investigación 
Cuando se contrastan los datos de matrícula internacional con el uso de Fan Pages como soporte de comunicación de marketing, es posible observar que, nuevamente, hay una correlación entre las universidades con mejores resultados de matrícula extranjera y el uso de Fan Pages en las acciones de Facebook. En contraste, las universidades menos exitosas en la atracción de estudiantes, tienden a conectarse con estudiantes internacionales mediante Perfiles y Grupos de Facebook (Cuadro 8).

Cuadro 8. Tipo de soporte utilizado, por cuartil y como porcentaje de las IES con presencia en Facebook

\begin{tabular}{|c|c|c|c|}
\hline & FAN & PERFIL & GRUPO \\
\hline Cuartil 1 & $40 \%$ & $40 \%$ & $20 \%$ \\
\hline Cuartil 2 & $23 \%$ & $54 \%$ & $23 \%$ \\
\hline Cuartil 3 & $69 \%$ & $19 \%$ & $13 \%$ \\
\hline Cuartil 4 & $76 \%$ & $12 \%$ & $12 \%$ \\
\hline
\end{tabular}

Fuente: Elaboración propia con datos de la investigación

Un análisis por país, muestra conductas similares. En Chile, $40 \%$ de las universidades del cuartil de mayor matrícula internacional usa Páginas de Facebook, un 10\% usa Perfiles y otro 10\% prefiere los Grupos. En cambio, un 17\% de las universidades del segmento de menor matrícula apoya sus esfuerzos en Perfiles. La situación se repite en porcentajes que descienden gradualmente en los demás segmentos.

En España en cambio, se produce una pequeña diferencia, aunque aún son los segmentos de mayor matrícula internacional los que usan Páginas de Facebook, los Perfiles y Grupos tienen mayor presencia en los segmentos intermedios de matrícula internacional, es decir, segundo y tercer cuartil.

Finalmente, en una visión general por país (Cuadro 9), en Chile el uso de Páginas de Facebook es más frecuente, solamente, entre las universidades de alta matrícula internacional, mientras que en las restantes, son los Perfiles los que acumulan mayor porcentaje de uso. En España por otro lado, los Fan Page son usados en mayor proporción por los cuartiles tercero y cuarto. El uso de Perfiles aparece ligeramente representado únicamente en el segundo cuartil de matrícula internacional. 
Cuadro 9. Tipo de acción en Facebook, como porcentaje del total de IES del cuartil. Por país

\begin{tabular}{|c|c|c|c|c|c|c|}
\hline & \multicolumn{3}{|c|}{ CHILE } & \multicolumn{3}{c|}{ ESPAÑA } \\
\hline & FAN & PERFIL & GRUPO & FAN & PERFIL & GRUPO \\
\hline Cuartil 1 & $6 \%$ & $17 \%$ & $6 \%$ & $21 \%$ & $7 \%$ & $7 \%$ \\
\hline Cuartil 2 & $7 \%$ & $14 \%$ & $0 \%$ & $11 \%$ & $26 \%$ & $16 \%$ \\
\hline Cuartil 3 & $8 \%$ & $8 \%$ & $0 \%$ & $50 \%$ & $10 \%$ & $10 \%$ \\
\hline Cuartil 4 & $40 \%$ & $10 \%$ & $10 \%$ & $39 \%$ & $4 \%$ & $4 \%$ \\
\hline
\end{tabular}

Fuente: Elaboración propia con datos de la investigación

\subsection{Desempeño de las páginas de Facebook}

Como hemos comentado en la primera parte de este artículo, las páginas de Facebook fueron lanzadas en 2007, específicamente orientadas al marketing de las compañías. La propia red social señala que: "ayudan a los negocios, organizaciones y marcas a compartir su historia y conectarse con el público". Estas páginas se definen, desde su génesis, como una herramienta de marketing y permiten a los usuarios recibir noticias acerca de la marca o la organización, directamente en sus secciones de noticias. (Facebook, 2015).

A continuación presentaremos los resultados del análisis del rendimiento de las páginas de Facebook de las instituciones que mantienen este tipo de soporte con propósitos de internacionalización, como hemos dicho, específicamente para la vinculación con estudiantes extranjeros. Vincularemos también estos rendimientos al número de estudiantes extranjeros matriculados en las instituciones, siguiendo la metodología de desagregación de las universidades por cuartiles.

Considerando únicamente las universidades chilenas presentes en Facebook, determinamos que el 25\% con mayor matrícula internacional usa Fan Pages, mientras solo un 6\% de las IES en los cuartiles siguientes lo usan. En España en cambio, las universidades de los dos cuartiles de mayor matrícula internacional, usan Fan Pages en un 25\% y 23\% respectivamente. Mientras, los cuartiles de menor matrícula internacional no alcanzan un 10\% de participación en este tipo de acción. Los resultados muestran que la constante permanece a lo largo del análisis, las universidades con mayor matrícula internacional presentes en la plataforma social, tienen mayor tendencia al uso de páginas de Facebook como herramienta de comunicación de marketing internacional.

En relación al desempeño de estas páginas, podemos ver, en el Cuadro 10, una primera aproximación a los comportamientos de los tres indicadores mencionados en la metodología, desagregados y consolidados por cuartil, es decir, en el caso del alcance, presentamos la suma total de los Tamaños de Comunidad de las universidades pertenecientes a cada 
grupo. En el caso de la frecuencia, presentamos la suma total de publicaciones diarias realizadas por las instituciones del cuartil desde el inicio de sus actividades en la página. Finalmente, la tasa de engagement, se presenta también como la suma de las tasas que individualmente consiguió cada universidad perteneciente al cuartil.

Cuadro 10. Desempeño de las páginas de Facebook, consolidado como el total de cada variable para las IES que pertenecen al cuartil

\begin{tabular}{|c|c|c|c|c|}
\hline PERFORMANCE & $\mathrm{N}^{\circ}$ IES & ALCANCE TOTAL & FRECUENCIA & ENGAGEMENT \\
\hline Cuartil 1 & 4 & 5021 & 2,890 & $9 \%$ \\
\hline Cuartil 2 & 3 & 10374 & 1,593 & $0 \%$ \\
\hline Cuartil 3 & 11 & 20401 & 5,810 & $16 \%$ \\
\hline Cuartil 4 & 13 & 34466 & 4,853 & $12 \%$ \\
\hline
\end{tabular}

Fuente: Elaboración propia con datos de la investigación

Como podemos ver, la columna “ $N^{\circ}$ IES" presenta el número de instituciones que usan Fan Page, correspondientes a cada cuartil, nuevamente vemos que las instituciones con mayor número de matriculados internacionales (cuartiles 3 y 4) son las que en mayor cantidad participan de este tipo de acción. Las siguientes columnas muestran los resultados acumulados de cada una de las métricas, y se puede observar que la tendencia parece continuar, es decir, los cuartiles con mayor número de estudiantes matriculados, poseen los mejores rendimientos acumulados.

Sin embargo, el número de universidades que publica material en Facebook mediante Fan Page, es diferente en cada cuartil, esto es, en los segmentos de mayor matrícula internacional, se agrupan un número muy superior de universidades que en los segmentos de menor matrícula. Frente a este dato, nos pareció necesario hacer una segunda lectura. Con objeto de tener una visión más clara del desempeño individual, presentaremos también los indicadores de resultado como promedio, es decir, el resultado combinado de cada variable en cada cuartil, pero esta vez como media.

En el Cuadro 11, se presentan las métricas de desempeño como promedio de las universidades del cuartil. Cuando observamos el Alcance como promedio de los tamaños de las comunidades de cada cuartil, vemos que el escenario ofrece una perspectiva distinta. Las 3 universidades pertenecientes al segundo cuartil promedian una comunidad superior al resto de las instituciones del sector, incluso sobre aquellas de mayor matrícula internacional. 
Cuadro 11. Alcance, Frecuencia y Engagement como promedio de las universidades por cuartil

\begin{tabular}{|c|c|c|c|c|}
\hline & $\mathrm{N}^{\circ}$ IES & ALCANCE Promedio & FRECUENCIA Promedio & ENGAGEMENT Promedio \\
\hline Cuartil 1 & 4 & 1255 & 0,72 & $2,3 \%$ \\
\hline Cuartil 2 & 3 & 3458 & 0,53 & $0,1 \%$ \\
\hline Cuartil 3 & 11 & 1855 & 0,53 & $1,5 \%$ \\
\hline Cuartil 4 & 13 & 2651 & 0,37 & $1,0 \%$ \\
\hline
\end{tabular}

Fuente: Elaboración propia con datos de la investigación

Situación similar se observa en el indicador "frecuencia", recordemos que la frecuencia es el promedio de publicaciones hechas diariamente; vemos nuevamente que los mayores índices no están en las universidades con mayor número de estudiantes extranjeros. En este caso, las universidades de menor matrícula internacional hicieron un promedio de 0,72 publicaciones por día, frente a las 0,37 del cuartil de mayor matrícula. Los cuartiles intermedios, que comparten el promedio de publicaciones, aun tuvieron mejor desempeño que el grupo de mayor matrícula.

Finalmente, en la cuarta columna presenta los datos relacionados con la tasa de Engagement, en cuyo caso, se mantiene la tendencia de las otras dos variables de desempeño, nuevamente, las universidades de menor matrícula fueron capaces de obtener un mayor promedio de interacciones con sus seguidores, completando el cuadro de desempeño y confirmando que aquellas instituciones que están en el grupo con menor cantidad de estudiantes, son las que tienen mejor desempeño en la gestión de sus páginas de Facebook.

Al contrario de los datos presentados respecto de la presencia en web y en Facebook, y el uso de Fan Page, en el caso de los desempeños, existe, al menos para España y Chile, una relación inversa entre el número de estudiantes extranjeros matriculados y el desempeño de las universidades en sus páginas de Facebook.

\section{Discusión}

El amplio uso de webs internacionales, muestra que las instituciones de ambos países han comprendido el valor de la comunicación web, se han hecho cargo de la necesidad de poner información a disposición de los receptores en plataformas de acceso expedito. Particularmente para los públicos de carácter internacional, que deben enfrentar la distancia como obstáculo para la búsqueda de información para seleccionar un destino internacional, este tipo de información es vital. Sin embargo, es prematuro asumir que la sola presencia de las universidades en la Web, aunque ventajosa, pudiera estar resolviendo el problema del contacto efectivo y la construcción de la reputación de las universidades en Chile y España. 
El mercado global de la educación superior, que se muestra altamente competitivo, está integrado por un gran número de universidades comunicando profusamente sus ventajas. Aquello y los cambios en las dinámicas de búsqueda y valoración de la información por parte de los stakeholders; permiten concluir, con cierta facilidad, que la presencia en web con contenido dirigido a segmentos internacionales, no es ya una condición discriminatoria, sino más bien, un requisito básico para la comunicación efectiva. Entonces, aunque difícilmente aporte la diferenciación necesaria, y menos aún una ventaja comparativa, para sobreponerse a otras universidades, será vital siquiera para existir en la mente de sus potenciales estudiantes internacionales.

Es destacable entonces, que los sistemas universitarios de España y Chile muestren una evidente tendencia a la difusión de información a estudiantes internacionales mediante el uso de sitios web específicos. Las universidades de ambos países mostraron interés en estas actividades para la difusión de información concerniente a sus procesos de internacionalización. España se observa una mayor penetración de este tipo de apartados web; casi la totalidad de las universidades de ese país usa estas herramientas.

Consistentemente, España mostró los mayores índices de matrícula de estudiantes extranjeros y la totalidad de universidades incluidas en los cuartiles de mayor de matrícula internacional mantiene este tipo de sitio; validando así, el uso de web internacionales como factor que favorece el éxito de la internacionalización, particularmente en la dimensión referida a la movilidad internacional de estudiantes.

En esa misma línea, las universidades chilenas parecen algo menos proclives a este tipo de sección, 42 de las 55 analizadas los tiene. No obstante, el $100 \%$ de las que registraron mayores índices de matrícula internacional los usa. Esa cifra fue decreciendo de manera proporcional al decrecimiento de estudiantes extranjeros matriculados.

Es posible constatar entonces, de acuerdo a los índices obtenidos en ambos países, que existe una relación entre la matrícula de estudiantes internacionales y el uso de este tipo de sitio web; la relación se presenta en una proporción directa, es decir, a mayor número de estudiantes extranjeros matriculados, mayor será la probabilidad de que la universidad estén usando este tipo de sitio con contenido internacional, y aunque no es posible asumir que la atracción de estudiantes sea resultado exclusivo de la existencia de estos espacios en la web, la evidente relación se constituye en un dato interesante para aquellas instituciones que aún mantienen el contacto con sus stakeholders internacionales a través de sitios institucionales, o simplemente fuera de Internet.

En este mismo sentido, una de las limitaciones de este estudio, se traduce justamente en la incapacidad de establecer si el número de estudiantes extranjeros matriculados es lo que impulsa a las universidades a construir sitios web específicos para comunicarse con ellos. O por el contrario, la presencia de estos sitios es la que contribuye al aumento de las cifras de matrícula internacional. 
Sin embargo, ayudará saber que múltiples estudios realizados sobre factores que determinan la movilidad internacional de estudiantes, particularmente aquellos referidos a la selección de un destino internacional, destacan aspectos vinculados el acceso a la información como factor clave en la toma de decisión. Ver por ejemplo (Beine, Noël y Ragot, 2014; González, Mesanza y Mariel, 2011; Llewellyn-Smith y McCabe, 2008; Mazzarol y Soutar, 2002; Moogan, Baron y Harris, 1999; Souto-Otero et al., 2013).

La participación de las universidades en Facebook no se mostró tan evidente como en el caso de los sitios internacionales. Sin embargo, fue posible observar que poco más de la mitad de las instituciones públicas españolas está presente en esa red social, mientras que, únicamente, el 39\% de las chilenas lo hace. En ambos países, las universidades públicas muestran mayor presencia, aunque la diferencia con las universidades privadas no fue estadísticamente significativa.

Una vez más, hay una relación directa entre el número de estudiantes extranjeros matriculados y la participación en Facebook. Un 52\% de las universidades con mayor matrícula internacional están presentes en Facebook, frente a solo un $31 \%$ de las que están en el cuartil más bajo de matrícula extranjera.

Aunque los resultados no muestran un uso mayoritario de Facebook como herramienta de comunicación de marketing entre las universidades, justamente aquellas que sí usan la plataforma pueden definirse como las más exitosas en sus procesos de atracción de estudiantes extranjeros. A pesar de que, nuevamente, estos datos no son suficientes para afirmar que exista una relación de causalidad única entre estos fenómenos, es decir, que la presencia en web asegure el éxito en la atracción de estudiantes internacionales; se puede ver que los responsables de la comunicación internacional al interior de las universidades han reconocido el valor de las acciones en la red social, y han apostado por desarrollar, de manera progresiva, acciones en ella.

Aparece entonces, como otra limitación de este estudio y a su vez, como un desafío interesante, avanzar en definir en estudios posteriores (tal vez más específicos y centrados en el valor que los estudiantes asignan a Facebook, como fuente de información para la decisión de un destino internacional), si esta tendencia en el uso de Facebook para la comunicación con stakeholders internacionales, impacta directamente en el aumento de los estudiantes extranjeros en una universidad; o si por el contrario, este aumento de la población extranjera entre los estudiantes, ha impulsado a las instituciones a entrar en Facebook como una alternativa para comunicarse con ellos más efectivamente.

Así como la presencia en la web, hoy día, ya no es una decisión determinante en el liderazgo y la diferenciación de las universidades; el uso de las redes sociales como plataformas de contacto con los públicos parece, en cambio, tener un carácter obligatorio. Las universidades no podrán elegir mantenerse al margen de este tipo de soporte, dado que, como hemos dicho, los estudiantes han emigrado a las redes sociales para la obtención de información. Las plataformas sociales como Facebook, se han convertido en una de las fuentes preferidas por los estudiantes para relacionarse con las instituciones y 
para conseguir información valiosa en el proceso de toma de decisión. Parece inevitable, entonces, que las universidades aumenten su presencia en las plataformas sociales.

La preferencia de páginas de Facebook, como herramienta con un carácter específico de marketing, frente a Perfiles y Grupos, nuevamente es más evidente en las universidades con más extranjeros, las universidades con mayor número de este tipo de alumnos usa Fan Pages, mientras que en el caso de los Perfiles, la mayor evidencia de su uso está en las universidades de menor matrícula extranjera.

Este comportamiento, parece estar mostrando que la presencia de las universidades en Facebook, no necesariamente revela una intención estratégica, sino más bien, una respuesta a la presión de entorno, una reacción a la suerte de moda reinante entre las organizaciones que se desenvuelven en entornos competitivos. Esta tendencia general, parece estar forzando a las universidades a aparecer en estas plataformas, incluso sin comprender con claridad su valor comunicacional y su impacto en los objetivos de marketing.

Los Perfiles y Grupos de Facebook, se comportan como herramientas sociales a un nivel personal, es decir, permiten la relación de las personas con su red de contactos, el intercambio de información, compartir contenidos y, en general, desarrollar una relación al interior de la red. Sin embargo, los Perfiles y Grupos, no ofrecen opciones de seguimiento y control, no permiten la evaluación del desempeño y, ciertamente, no apuntan al cumplimiento de objetivos comunicacionales o de marketing.

Estos antecedentes demuestran, probablemente, que quienes están tomando estas decisiones, lo hacen desde una mirada operativa, de reacción, y no con un fin estratégico. En este escenario, nos podríamos plantear un par de situaciones que debieran ser consideradas en la comprensión de este fenómeno. Primero, que las universidades podrían no estar enfrentando su proceso de comunicación internacional con una visión estratégica, es decir, podría ser que sus acciones estén implementándose de manera reactiva y a nivel operativo, y no apuntadas a conseguir los objetivos institucionales.

En segundo lugar, este comportamiento podría estar entregando señales respecto de quienes están tomando las decisiones de comunicación internacional en las universidades. El uso de Facebook mediante Perfiles y Grupos, denota la ausencia de conocimiento de la herramienta y sus efectos en la estrategia de marketing, y por tanto, podríamos suponer que los responsables de su implementación carecen de conocimientos respecto de ella, o lo que sería aún más complejo, carecen de conocimientos suficientes en comunicación o marketing.

De este modo, se desvela otra pregunta para futuras indagaciones en este campo: ¿̨cuál es el perfil profesional de los responsables de la comunicación internacional en las universidades chilenas y españolas?

El desempeño de las universidades que sí usaron Fan Pages, presentó un comportamiento distinto al que, por ejemplo, se venía dando en relación a la presencia en web. Es decir, la existencia de una relación directa entre el número de estudian- 
tes extranjeros matriculados y el uso de estos soportes. En relación al desempeño en las páginas de Facebook, el estudio permitió comprobar que fue mejor en las universidades con menos estudiantes extranjeros.

El alcance promedio combinado del $50 \%$ de las universidades con menos extranjeros (cuartiles 1 y 2) fue ligeramente inferior al de las con mayor matrícula internacional. Considerando que el número de universidades en estos cuartiles fue 7 , frente a las 24 de los cuartiles superiores, es evidente que en esa variable, las universidades con menos alumnos extranjeros han conseguido mayores tamaños en sus comunidades. Este dato presenta una visión que podría cambiar la percepción respecto al modo en que las universidades con menor número de estudiantes extranjeros, están conduciendo su estrategia de marketing en social media.

El tamaño de la comunidad es una variable que se asocia directamente a la comunicación boca-oído (WOM, Word of mouth); además, las personas que se hacen Fan de una organización, están más comprometidas con la marca (Wallace et al., 2014) y gastan 5 veces más en la marca que los usuarios que no son Fan (Hollis, 2014), igualmente los Fans suelen tener 2.4 veces más amigos que un usuario normal de Facebook y hacen 5,3 veces más clic en enlaces externos, de modo que la cantidad de Fans de una organización se convierte en una efectiva forma de atraer usuarios bien conectados en la redes sociales y por tanto, ampliar el alcance del mensaje de las universidades. Para la comunicación universitaria internacional, la difusión de la información de manera informal, o por pares, como se ha mencionado antes, es sustantiva, pues aumentará las probabilidades de que esos mensajes alcancen efectivamente a sus receptores. Y parece ser que las universidades pequeñas (en función de su matrícula internacional), lo han comprendido.

Cuando se observa la frecuencia promedio, la situación se repite de manera más evidente aun. Las universidades del primer cuartil fueron capaces de publicar contenido un $51 \%$ más frecuentemente que las del cuartil de mayor matrícula internacional. Este factor de desempeño es nuevamente muy importante en la comunicación mediante redes sociales, y más aún en entornos de alta competencia, como es el mercado global de la educación superior, pues la gran cantidad de información vertida por cada institución y la gran cantidad de instituciones que están comunicando activamente, hace que las opciones reales de alcanzar a los públicos, con un mensaje determinado, disminuya significativamente si ese mensaje no está constantemente expuesto para sus receptores.

En este sentido, altas frecuencias, denotan una visión estratégica del uso de los Fan Page, o al menos una comprensión más profunda del modo en que circula la información en ellos, y sobre todo, el modo en que los usuarios la consumen.

Finalmente en la tasa de engagement, el comportamiento se mantiene, las universidades con más extranjeros, consiguieron tasas promedio de 1,0\%, mientras que las de menor matrícula, alcanzaron tasas del 2,3\% promedio. 
En resumen, en el caso de España y Chile, las universidades con menos alumnos extranjeros están teniendo mejores desempeños en el uso de páginas de Facebook como herramientas de Marketing, mientras que las de mayor matrícula, aunque con mejor presencia, consiguen rendimientos menores.

Estos resultados, nos hacen pensar nuevamente lo expuesto con anterioridad; al parecer, los cuartiles de mayor matrícula, integrados por las grandes universidades de cada país, es decir aquellas con mayor notoriedad y con una reputación suficientemente sólida, podrían estar teniendo estos menores desempeños por estar simplemente reaccionando a las presiones del entorno, pero no estar usando esa herramienta desde una lógica estratégica, o con objetivos claros y definidos.

En cambio, las pequeñas universidades, que requieren de mayores esfuerzos para alcanzar sus metas de internacionalización o atracción de estudiantes, podrían haber encontrado, en las redes sociales, una oportunidad de alcanzar con mayor efectividad a sus públicos, y en consecuencia, podrían estar usándolas efectivamente, con un fin concreto de marketing. Por supuesto queda aún por determinar un sin número de factores que pudieran explicar más profundamente estas conductas. Sin embargo, el presente estudio aporta un punto de partida para el análisis más amplio del uso de Fan Pages como herramientas de marketing internacional en las universidades.

Creemos que el análisis de los impactos concretos del uso de las plataformas sociales en la atracción de estudiantes, así como la revisión de factores estructurales y de gestión, como la disponibilidad presupuestaria, la reputación de las universidades, la estructura estratégica del trabajo de marketing internacional, la conciencia del uso de las herramientas de comunicación en redes sociales y el perfil profesional de los responsables de comunicación internacional; son temas que podrían motivar futuras investigaciones, que aporten luces más claras respecto a los beneficios de este tipo de prácticas y sus impactos en la atracción de estudiantes extranjeros, como parte del éxito de los procesos de internacionalización universitaria.

\section{Referencias bibliográficas}

Alzamel, S. (2014): "Factors that Influence Student Satisfaction with International Programs in Institutions of Higher Learning: A Proposed Case Study of University of Dayton”, International Journal of Global Business, n. 7 (1), pp. 15-24.

Beine, M., Noël, R., y Ragot, L. (2014): “Determinants of the international mobility of students”, Economics of Education Review, n. 41, pp. 40-54. doi:10.1016/j.econedurev.2014.03.003

Boyd, D. M., y Ellison, N. B. (2007): "Social network sites: Definition, history, and scholarship", Journal of Computer-Mediated Communication, n. 13 (1), pp. 210-230.

Burson-Marsteller (2012): Global Social Media Check Up 2012 Presentation, http://www.burson-marsteller.com/social/Documents/Burson-Marsteller Global Social Media Check-Up 2012.pdf, [consultado el 08/04/2015].

74 | n 21, pp. 55-77 | doxa.comunicación 
Carpenter, C., y Drezner, D. W. (2010): "International Relations 2.0: The implications of new media for an old profession", International Studies Perspectives, n. 11(3), pp. 255-272. doi:10.1111/j.1528-3585.2010.00407.x

Chapleo, C., Carrillo, M., y Castillo, A. (2011): "Do UK universities communicate their brands effectively through their websites?”, Journal of Marketing for Higher Education, n. 21(1), pp. 37-41. doi:10.1080/08841241.2011.569589

Colliander, J. (2012): Socially Acceptable? Exploring Consumer Responses to Marketing in Social Media. Estocolmo: Stocholm School of Economics, http://www.diva-portal.org/smash/get/diva2:570413/FULLTEXT01.pdf, [consultado el 08/04/2015].

Djerasimovic, S. (2014): "Examining the discourses of cross-cultural communication in transnational higher education: from imposition to transformation", Journal of Education for Teaching, n. 40, pp. 204-216. doi:10.1080/02607 476.2014 .903022

Facebook. (2015): Conceptos básicos de las páginas, https://www.facebook.com/help/281592001947683/, [consultado el $07 / 01 / 2015]$.

Fagerstrøm, A., y Ghinea, G. (2013): "Co-creation of value in higher education: using social network marketing in the recruitment of students", Journal of Higher Education Policy and Management, n. 35(1), pp. 45-53. doi:10.1080/ 1360080X.2013.748524

Gacel-Ávila, J. (2012): Comprehensive Internationalisation in Latin America, Higher Education Policy, n. 25(4), pp. 493510. doi:10.1057/hep.2012.9

Gobierno de Chile. (2014a): Directorio Instituciones de Educación Superior. http://divesup.cl/index2.php?id_portal=38\& id_seccion=3250\&id_contenido=13212, [consultado el 04/11/2014].

Gobierno de Chile. (2014b): División de Educación Superior, http://divesup.cl/index2.php?id_portal=38\&id_seccion=3250\&id_contenido=13212, [consultado el 22/12/2012].

Gobierno de España. (2014): Las cifras de la educación en España. Curso 2011-2012 (Edición 2014), http://www.mecd.gob.es/servicios-al-ciudadano-mecd/estadisticas/educacion/indicadores-publicacionessintesis/cifras-educacion-espana/2014.html, [consultado el 12/12/2014].

Gobierno de España. (2015): Universidades de España, http://www.mecd.gob.es/educacion-mecd/areaseducacion/universidades/educacion-superior-universitaria/que-estudiar-donde.html, [consultado el 21/12/2014].

González, C. R., Mesanza, R. B., y Mariel, P. (2011): “The determinants of international student mobility flows: An empirical study on the Erasmus programme”, Higher Education, n. 62(4), pp. 413-430. doi:10.1007/s10734-010-9396-5

González-molina, S., y Ramos, F. (2014): "Las redes sociales en el ámbito periodístico: i cómo usan los medios europeos de referencia sus perfiles en Twitter y Social Media”, Comunicación y Hombre, n. 10(1), pp. 37-52.

Gordan, C., y Pop, M. (2013): “Incorporating Market Orientation in Highier Education Institutions”, Annals of the University of Oradea, Economic Science Series, n. 22(1), pp.1743-1753. 
Grönroos, C. (2008): “Service logic revisited: who creates value? And who co-creates?", European Business Review, n. 20(4), pp. 298-314. doi:10.1108/09555340810886585

Hennig-Thurau, T., Malthouse, E. C., Friege, C., Gensler, S., Lobschat, L., Rangaswamy, A., y Skiera, B. (2010): “The Impact of New Media on Customer Relationships", Journal of Service Research, n. 13(3), pp. 311-330. doi:10.1177/ 1094670510375460

Hollis, N. (2014): The value of a social media fan, http://www.millwardbrown.com/global-navigation/blogs/post/mbblog/2011/04/04/The-value-of-a-social-media-Fan.aspx, [consultado el 11/04/2015]

Kaplan, R. S., y Norton, D. P. (2007): “Using the balanced scorecard as a strategic management system”, Harvard Business Review, n. 85(7-8), pp.150-161. doi:10.1108/10878570410699825

Kaushik, A. (2010): Web Analytics 2.0: The Art of Online Accountability and Science of Customer Centricity. Indianapolis: John Wiley \& Sons.

Knight, J. (2013): “Education hubs: international, regional and local dimensions of scale and scope," Comparative Education, n. 49(3), pp. 374-387. doi:10.1080/03050068.2013.803783

Lee, D., Hosanagar, K., y Nair, H. S. (2014): The Effect of Social Media Marketing Content on Consumer Engagement: Evidence from Facebook, ISIS Conference.

Llewellyn-Smith, C., y McCabe, V. S. (2008): "What is the attraction for exchange students: The host destination or host university? Empirical evidence from a study of an Australian University", International Journal of Tourism Research, n. 10(6), pp.593-607. doi:10.1002/jtr.692

Maringe, F, y Carter, S. (2007): “International students' motivations for studying in UK HE: insights into the choice and decision making of African students", International Journal of Educational Management, n. 21(6), pp. 459-475. doi:10.1108/09513540710780000

Mashable Social Media. (2011): How Non-Profits Are Using Social Media, http://mashable.com/2011/10/14/how-nonprofits-use-social-media/, [consultado el 08/04/2015].

Mazzarol, T., y Soutar, G. N. (2002): "Push-pull' factors influencing international student destination choice”, International Journal of Educational Management, n. 16(2), pp. 82-90. doi:10.1108/09513540210418403

Moogan, Y. J., Baron, S., y Harris, K. (1999): “Decision-Making Behaviour of Potential Higher Education Students”, Higher Education Quarterly, n. 53(3), pp. 211-228. doi:10.1111/1468-2273.00127

Murdough, C. (2010): “Social media measurement; It’s not impossible”, Journal of Interactive Advertising, n. 10(1), pp. 9499.

O'Connor, L., y Lundstrom, K. (2011): “The Impact of Social Marketing Strategies on the Information Seeking Behaviors of College Students”, Reference \& User Services Quarterly, n. 50(4), pp. 351-366. 
Pinto, M., Guerrero Quesada, D., y Granell, X. (2014): “Dissemination of information and visibility of the European Higher Education Area through the websites of Spanish universities: A longitudinal metric analysis, 2007-2012", Scientometrics, n. 98(2), pp. 1235-1255. doi:10.1007/s11192-013-1062-0

Reina, J., Fernández, I., y Noguer, Á. (2012): "El uso de las redes sociales en las universidades andaluzas: el caso de Facebook y Twitter”, Revista Internacional de Relaciones Públicas, n. II(4), pp. 123-144. doi:10.5783/RIRP-4-2012-06-123-144

Ross, M., Grace, D., y Shao, W. (2012): “Come on higher ed ... get with the programme! A study of market orientation in international student recruitment”, Educational Review, n. 65(2), pp. 1-22. doi:10.1080/00131911.2012.659656

Souto-Otero, M., Huisman, J., Beerkens, M., de Wit, H., Vujić, S., y VujiC, S. (2013): "Barriers to international student mobility: Evidence from the ERASMUS program”, Educational Researcher, n. 42(2), pp. 70-77. doi:10.3102/0013189X12466696

Thune, T., y Welle-Strand, A. (2005): "ICT for and in internationalization processes: A business school case study", Higher Education, n. 50(4), pp. 593-611. doi:10.1007/s10734-004-6368-7

Tofallis, C. (2012): “A different approach to university rankings", Higher Education, n. 63(1), pp. 1-18. doi:10.1007/s10734011-9417-z

Tomše, D., y Snoj, B. (2014): “Marketing Communication on Social Networks - Solution in the Times of Crisis", Marketing, n. 45(2), pp. 131-139.

Wallace, E., Buil, I., de Chernatony, L., y Hogan, M. (2014): “Who "likes” you...and why? A typology of facebook fans: From "fan"-atics and self- expressives to utilitarians and authentics", Journal of Advertising Research, n. 54(1), pp. 92-109. doi:10.2501/JAR-54-1-092-109

Wei, H. (2012): "An empirical study on the determinants of international student mobility: a global perspective", Higher Education, n. 66(19), pp. 105-122. doi:10.1007/s10734-012-9593-5

Wilcox, G. B., y Kim, K. K. (2013): “Measurement of social media effects: Social media performance model”, American Academy of Advertising. Conference Proceedings, 65.

- (2012): "Multivariate Time Series Use for the Measurement of Social Media Effects", Marketing Management Journal, n. 22(2), pp. 90-101.

Wilkins, S., Balakrishnan, M. S., y Huisman, J. (2012): "Student choice in higher education: Motivations for choosing to study at an international branch campus", Journal of Studies in International Education, n. 16(5), pp. 413-433. doi:10.1177/1028315311429002 